\title{
Retinal telangiectasis in adults: fluorescein angiographic findings and treatment by argon laser
}

\author{
AMRESH CHOPDAR \\ From the Fundus Clinic, Moorfields Eye Hospital, High Holborn, London
}

SUMMARY This article describes 3 cases of retinal telangiectasia, all presenting in middle age, affecting the macula. They have been investigated by means of fluorescein angiography and successfully treated by argon laser.

Telangiectasia involving the macula area alone is a rare congenital fundus disorder, which may remain undiscovered until late adult life (Reese, 1956; Henkind and Morgan, 1966). The disease can be suspected either on routine ophthalmic examination or when symptoms arise due to decompensation. The purpose of this paper is to analyse 3 such cases seen at the Fundus Clinic at Moorfields Eye Hospital, High Holborn, London, and to discuss their management.

\section{Case reports}

\section{CASE 1}

A 61-year-old man who gave a 3-year history of blurred vision in his right eye. He had had a partial gastrectomy and colostomy 2 years previously for a gastric neoplasm; otherwise he was in excellent physical health. His right visual acuity was $6 / 36$ and left 6/6. External slit-lamp examination of both eyes was normal. The right fundus showed macular oedema, spreading laterally, surrounded by a ring of hard exudate. Several red dots resembling microaneurysms were noted in this area. Fluorescein angiography showed tortuous dilated capillaries with microaneurysm formation extending laterally from the perifoveal arcade, and leakage of dye spreading to the macula in a typical cystoid manner. The left eye was normal.

The right eye was treated with the argon laser. Now after 2 years the visual acuity has improved to $6 / 12$, though the fluorescein angiogram still shows some residual malformation and leakage. Further photocoagulation would be necessary to achieve full control of the leakage, but it would need to be

Address for reprints: A. Chopdar, FRCS, Moorfields Eye Hospital, High Holborn, London WC1V 7AN extensive, with the risk of reducing his present level of central vision, so the case remains under observation (Fig. 1).

CASE 2

A 55-year-old man was seen with an 8-month history of metamorphopsia in his right eye. The visual acuities were $6 / 24$ in the right eye and 6/6 in the left eye. The external examination, including biomicroscopy, was normal. The right fundus showed an area of serous detachment of the macula with a ring of hard exudate laterally. Several discrete red dots, in appearance similar to microaneurysms, were noted at this site. The left fundus showed only a minimal degree of neurosensory separation of the macula, with a few red dots below and lateral to the macula. Fluorescein angiography of the right eye showed a typical telangiectatic malformation of the retinal capillaries with extensive leakage of dye, spreading to the macula. The left angiogram showed 2 groups of similar vascular malformations below and lateral to the macula, with late leakage. Both eyes were treated with the argon laser. Now after 2 years both fundi seem to be free from macular oedema, but fluorescein angiography shows minimal leakage of dye. The right vision has improved to $6 / 12$ and the left has been retained at 6/6 (Fig. 2).

CASE 3

A 45-year-old man with sound health was seen with a 1-year history of intermittent blurring of left vision. The right vision was $6 / 5$ and the left $6 / 12$. external examination of the eyes showed nothing remarkable. The right fundus was normal, but the left showed retinal oedema lateral to the macula, with mild vascular tortuosity and microaneurysms. Fluorescein angiography showed capillary abnormality, microaneurysms, and late leakage spreading 


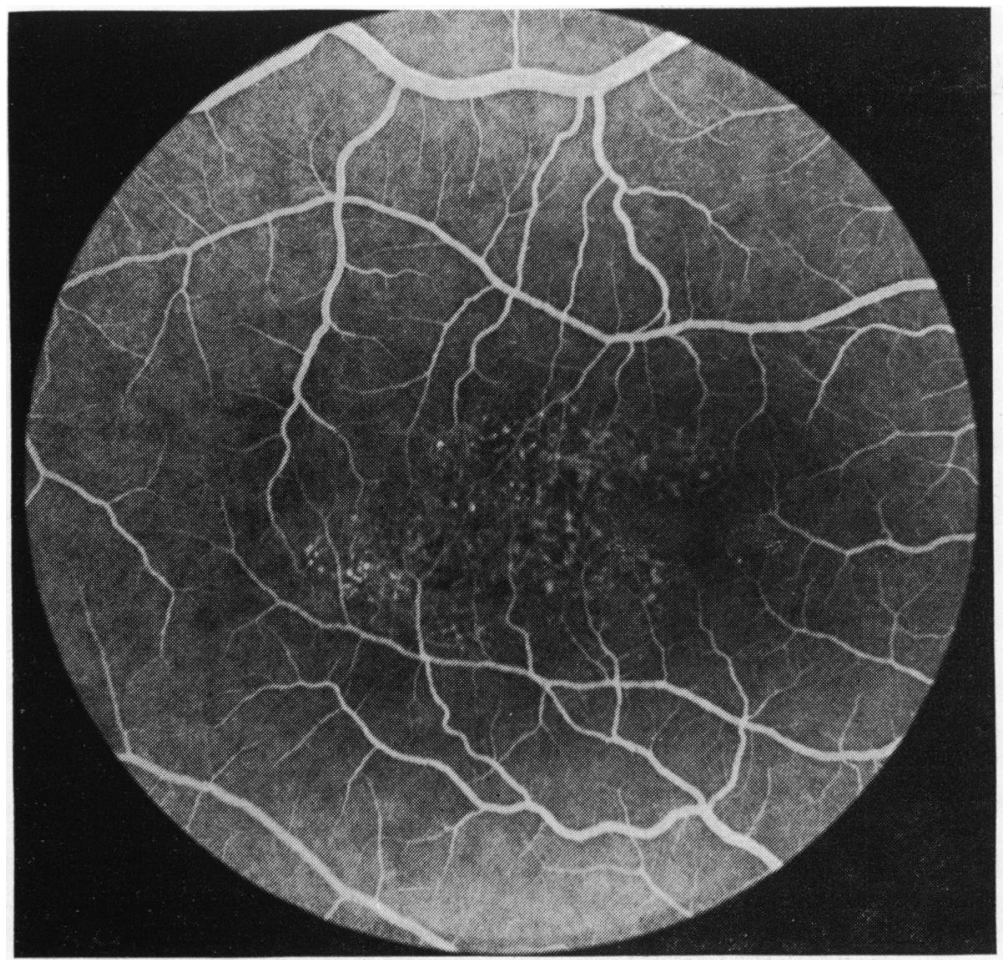

Fig. 1 (A) Pretreatment fluorescein angiogram of the right eye during the arteriovenous phase, showing marked capillary abnormalities with aneurysmal dilatation lateral to the macula

Fig. 1 (B) Late phase angiogram of the same eye showing leakage in a typical cystoid manner

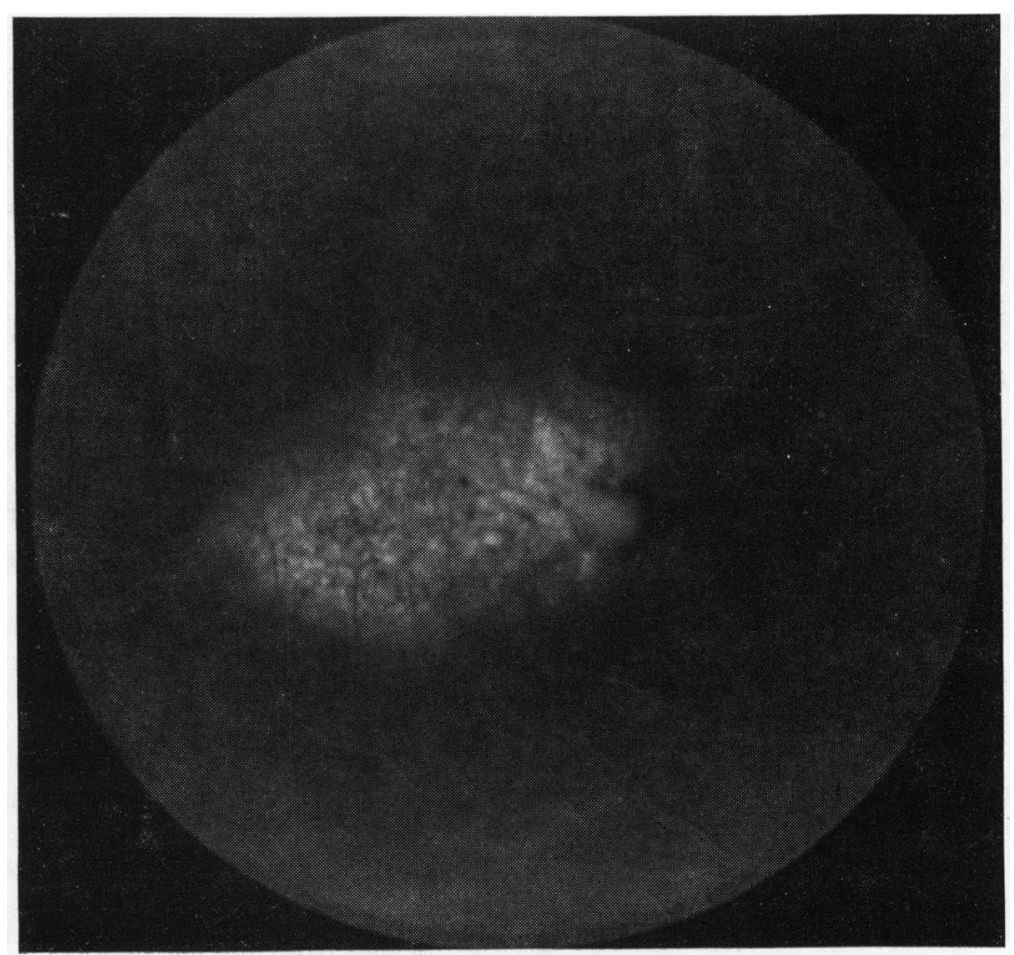


Fig. 1 (C) Post-treatment fuorescein angiogram of the same eye during the arteriovenous phase showing a minimal degree of residual vascular abnormalities round the perifoveal arcade

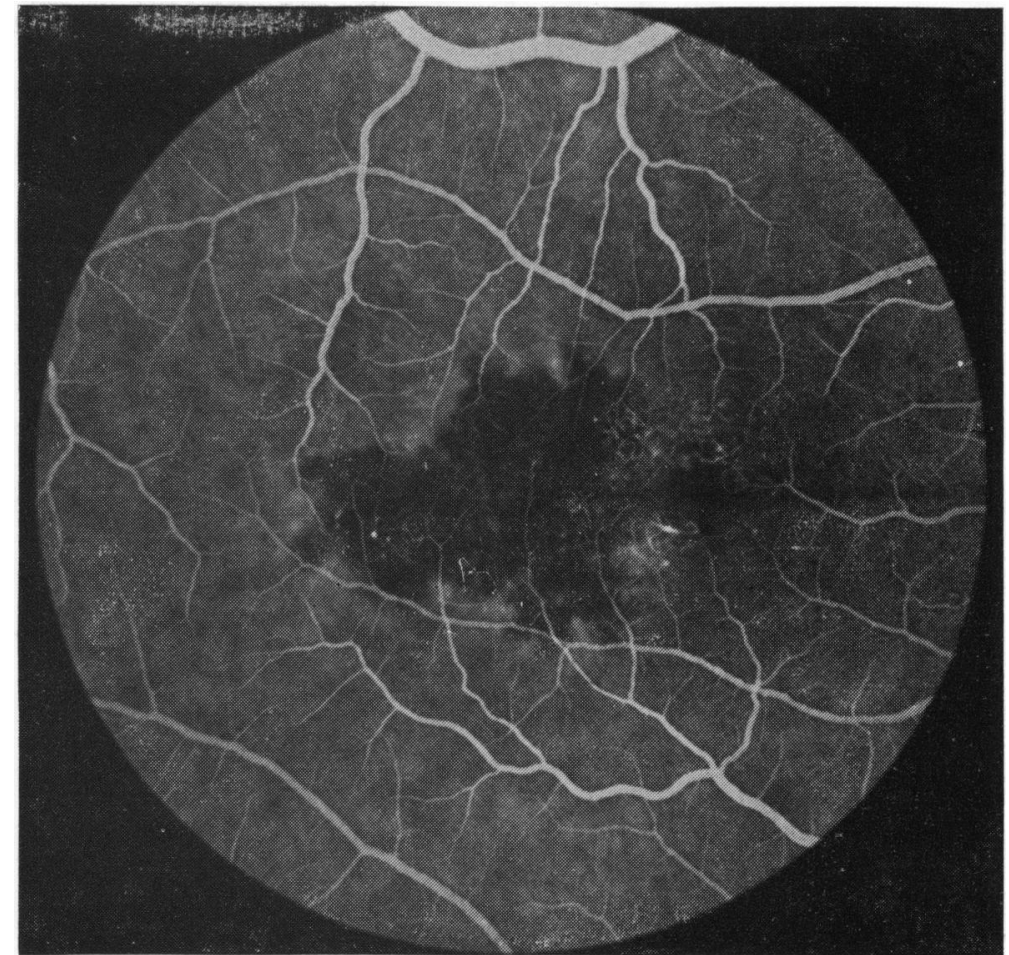

Fig. 1 (D) Late phase angiogram of the same eye showing minimal leak 


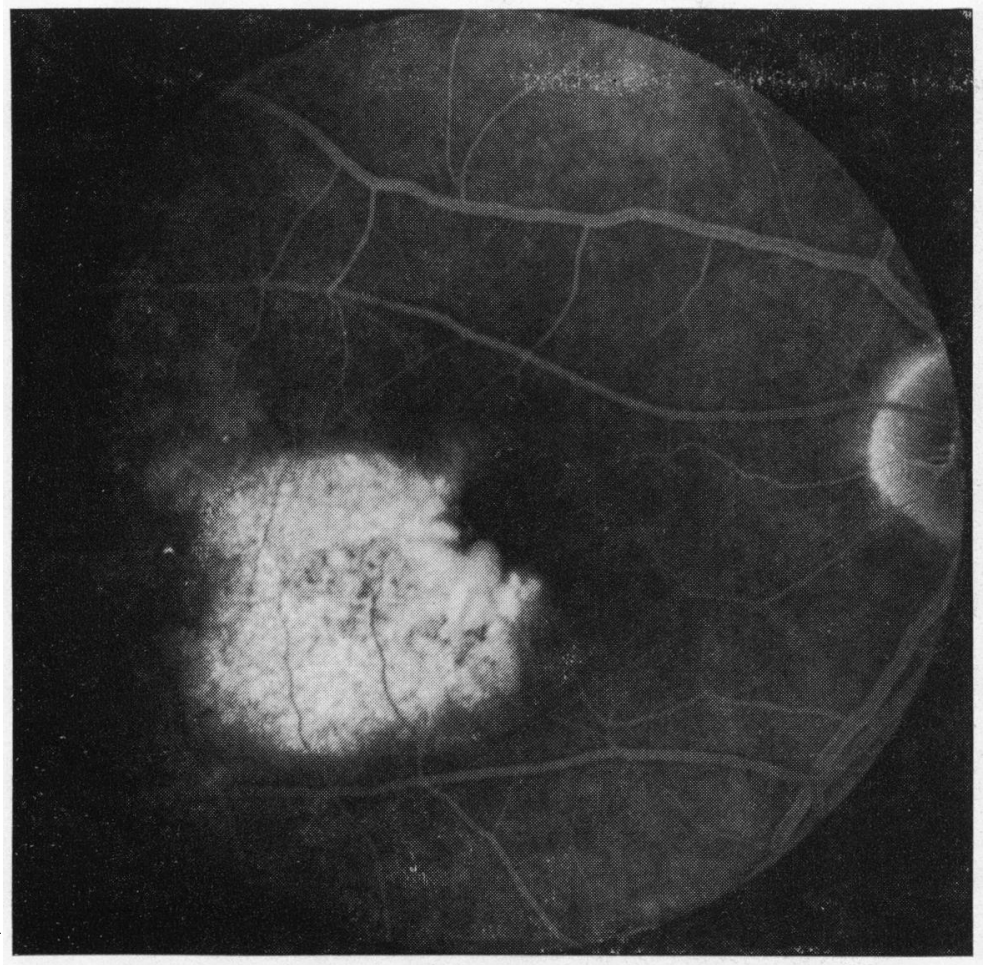

Fig. 2 (A) Pretreatment fluorescein angiogram of the right eye during the late phase showing extensive leak to the macula
Fig. 2 (B) Same eye after treatment during the late phase of the angiography shows minimal leakage

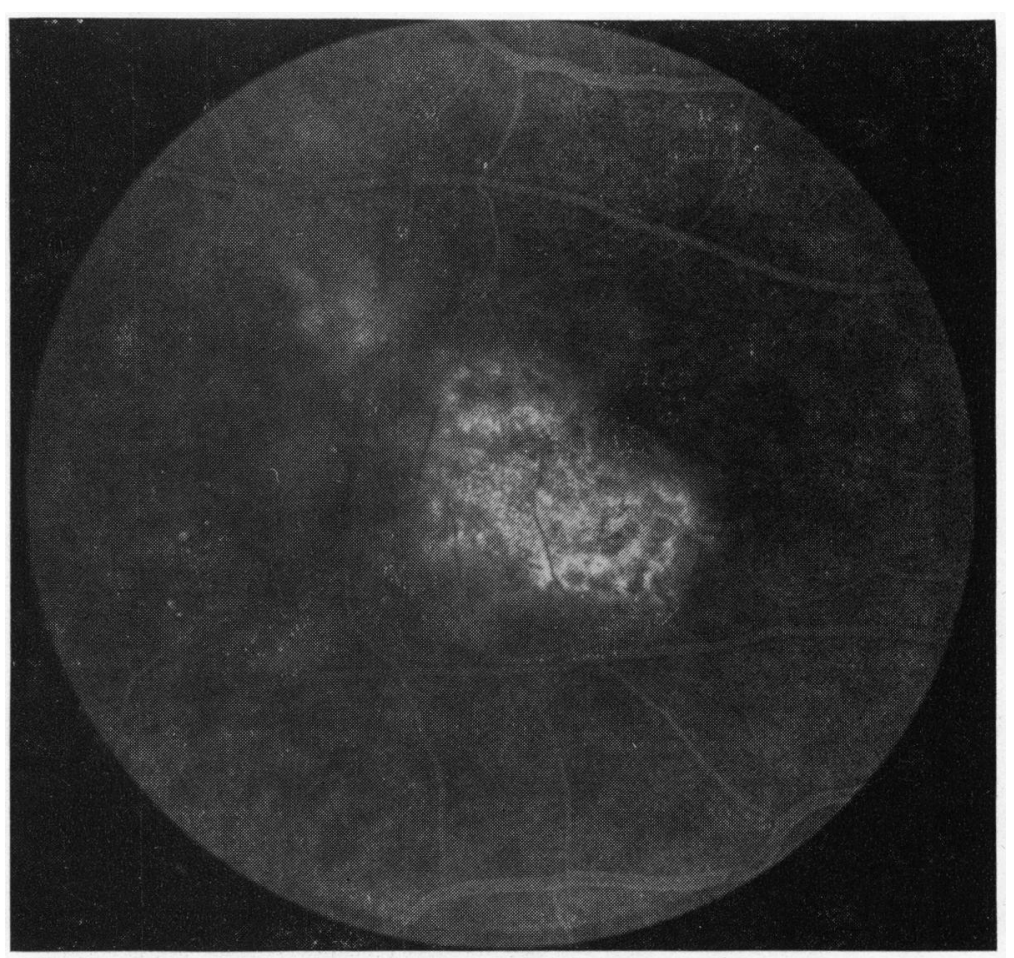


Fig. 2 (C) Pretreatment angiogram of the left eye showing late leakage

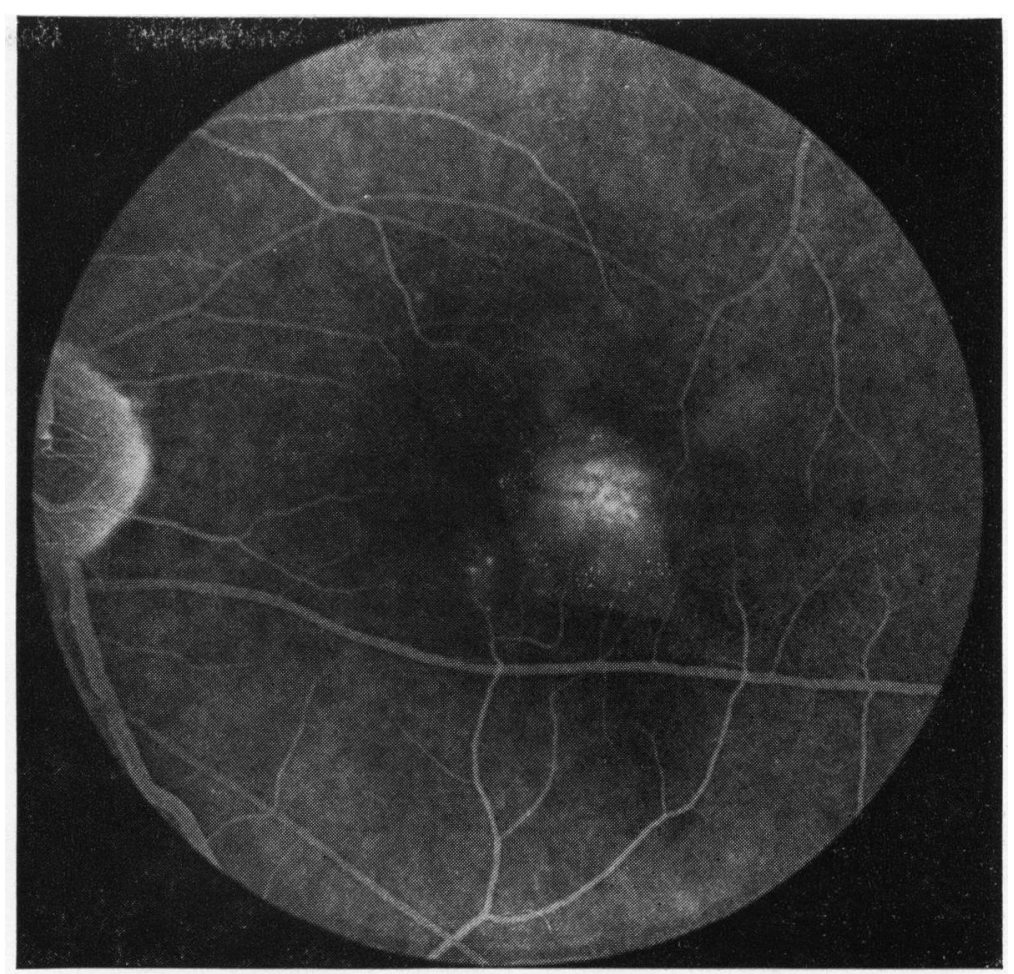

Fig. 2 (D) Same eye after treatment, with minimal leakage 


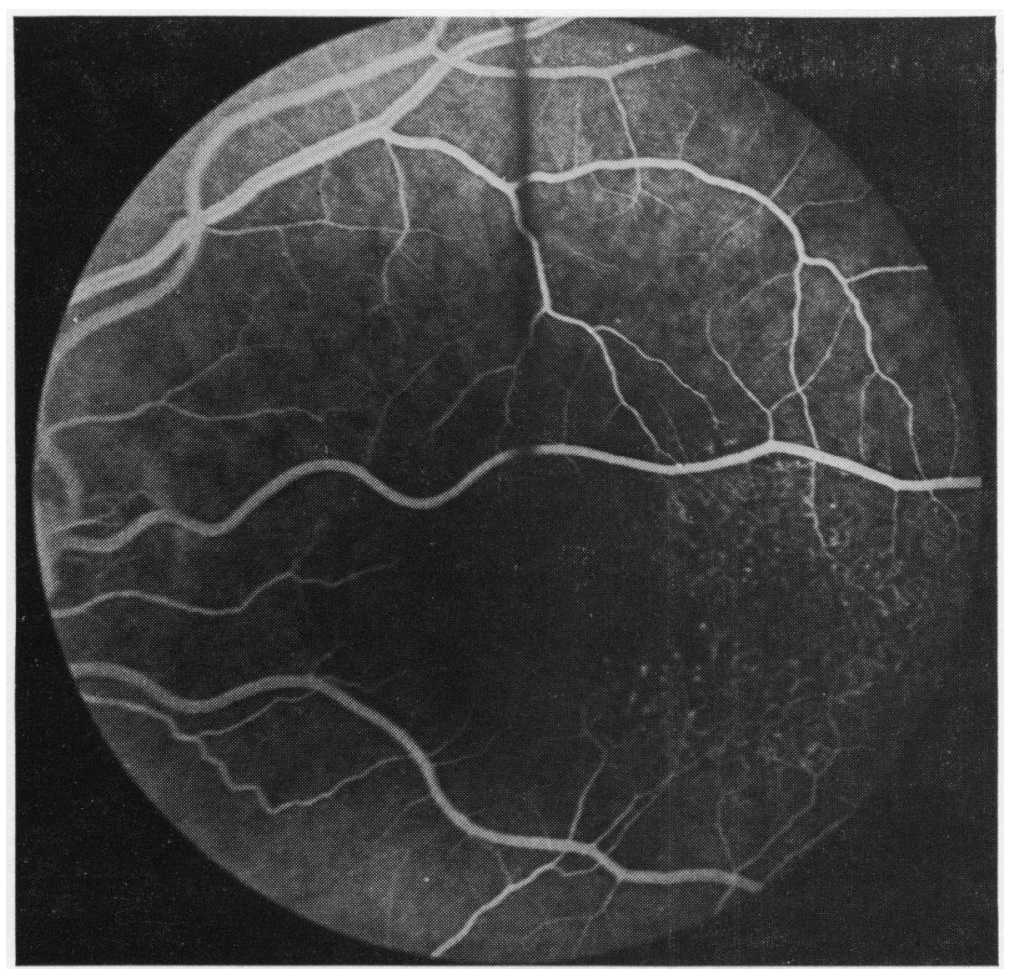

Fig. 3 (A) Fluorescein angiogram of the left eye during the arteriovenous phase showing capillary abnormality and aneurysmal formation lateral to the macula

Fig. 3 (B) Same eye 15 minutes after the injection of dye showing extensive leak

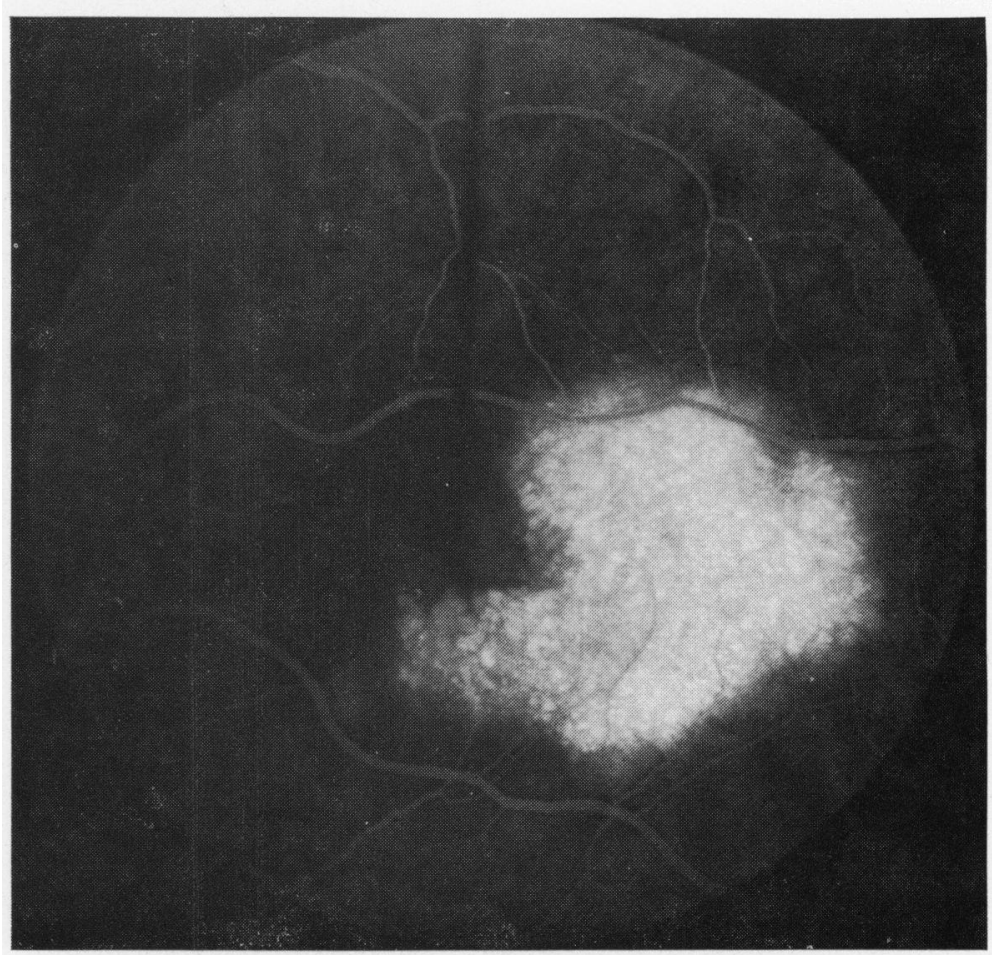


Fig. 3 (C) Post-treatment angiogram of the same eye during the arteriovenous phase showing no vascular abnormalities
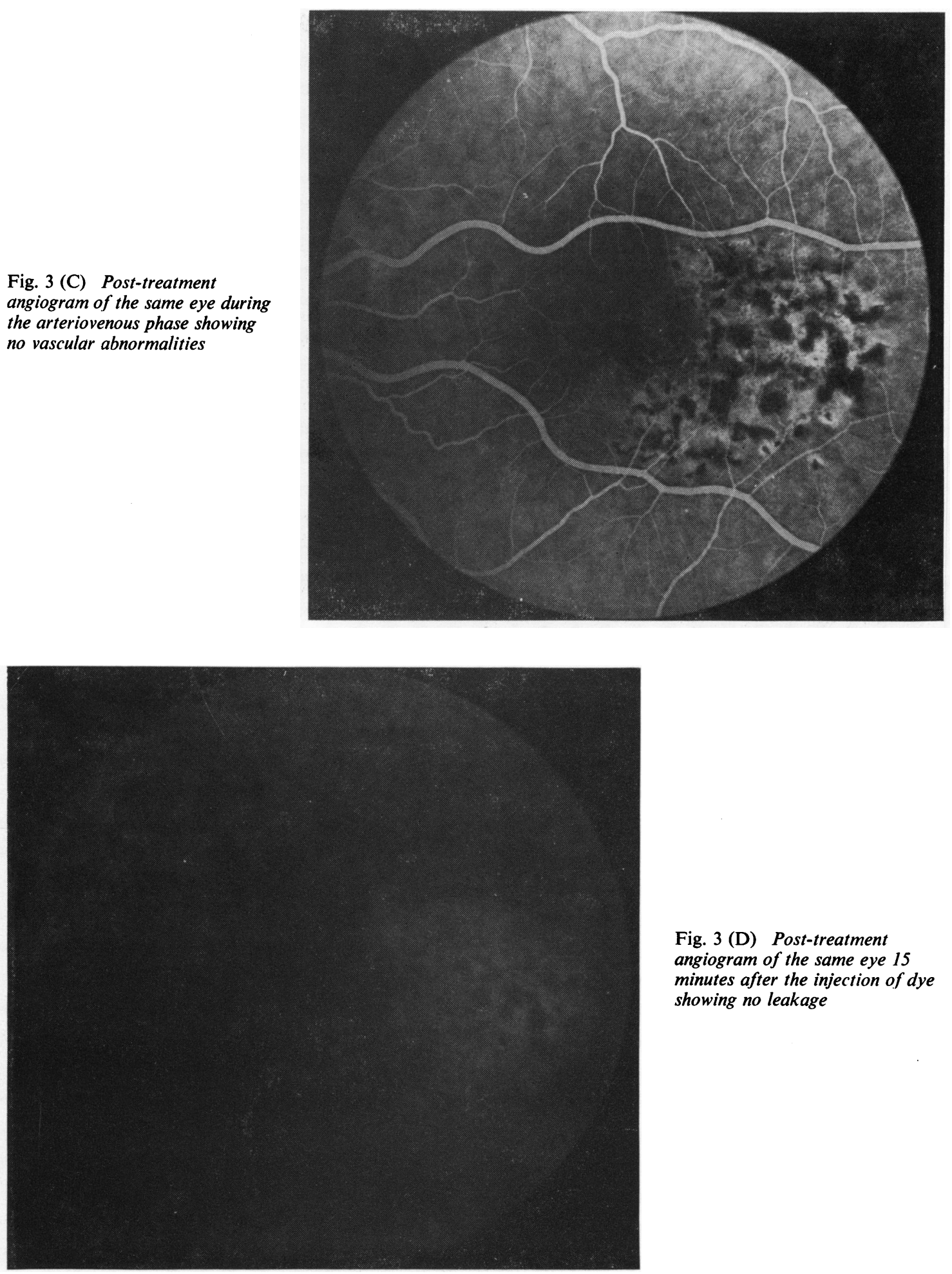

Fig. 3 (D) Post-treatment angiogram of the same eye 15 minutes after the injection of dye showing no leakage 
to the macula. This eye was treated with argon laser photocoagulation. Now 18 months later the left vision has improved to $6 / 7 \cdot 5$ (Fig. 3).

\section{Discussion}

All patients in this series were male and only 1 had bilateral disease. Their ages varied from 45 to 61 years (mean 53.7). There was no systemic vascular or metabolic disease that could suggest secondary involvement. Symptoms such as blurring of vision and metamorphopsia were mild and intermittent, so that the patients took 8 to 36 months before seeking advice (mean 18.7). All had a similar clinical picture with capillary abnormalities, microaneurysms, and a surrounding ring of hard exudate lateral to the macula. None of these patients showed any peripheral retinal disease. Fluorescein angiography showed the extent of the abnormality clearly, which was essential before laser treatment. The treatment had to be repeated on some occasions. Visual acuity improved in all cases.

Published reports contain many references to Coats's disease, miliary aneurysms of Leber, and angiomatosis retinae, but few to telangiectasia. Most authors describe lesions affecting the peripheral parts of the retina, but Reese (1956) has described cases affecting the macula and commented that telangiectasia could affect any part of the retina, including the optic disc. He suggested that Coats's disease and Leber's miliary aneurysms were one and the same condition but in different stages of evolution. Both together he called telangiectasia. Although the treatment of the peripheral and some of the posterior lesions has been possible with cryopexy and xenon-arc photocoagulation, the macular area remained a difficult problem until the availability of the argon laser, which undoubtedly gives a greater degree of accuracy in the treatment of these lesions and reduces the risk to the central vision. It is suggested that argon laser should be the treatment of choice in such cases.

I thank Professor David W. Hill for his helpful criticism and permission to report these cases under his care. I also thank Mrs Ann Marie Turk for the illustrations and Mrs Joyce Preen for secretarial help.

\section{References}

Henkind, P., and Morgan, G. (1966). Peripheral retinal angioma with exudative retinopathy in adults (Coats's lesion). British Journal of Ophthalmology, 50, 2-11.

Reese, A. B. (1956). Telangiectasis of the retina and Coats's disease. American Journal of Ophthalmology, 42, 1-8. 